\title{
Serum and peritoneal fluid concentrations of soluble human leukocyte antigen, tumor necrosis factor alpha and interleukin 10 in patients with selected ovarian pathologies
}

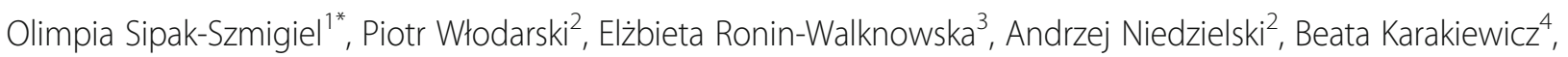
Sylwia Słuczanowska-Głąbowska ${ }^{5}$, Maria Laszczyńska ${ }^{6}$ and Witold Malinowski ${ }^{1}$

\begin{abstract}
Background: Although immune system plays a key role in the pathogenesis of both endometriosis and ovarian cancer, its function is different. Therefore, we hypothesized, that selected immune parameters can serve as diagnostic markers of these two conditions. The aim of this study was to compare serum and peritoneal fluid concentrations of sHLA-G, IL-10 and TNF-alpha in women with selected ovarian pathologies: benign serous cysts, endometrioma and malignant tumors. Clinical significance of using them for diagnostic purposes in women with serous ovarian cysts, endometriosis, and ovarian cancer, which in the future may improve the early diagnosis of ovarian diseases.
\end{abstract}

Case Presentation: The study included women treated surgically for benign serous ovarian cysts, ovarian endometrioma and serous ovarian adenocarcinomas. Peripheral blood and peritoneal fluid samples were obtained intraoperatively.

Patients with benign serous cysts, endometrioma and ovarian malignancies did not differ significantly in terms of their serum and peritoneal fluid concentrations of sHLA-G. Ovarian cancer patients presented with significantly higher median serum concentrations of IL-10 and TNF-alpha than other study subjects. Median concentrations of IL-10 and TNF-alpha in peritoneal fluid turned out to be the highest in ovarian cancer patients, followed by women with endometrioma and subjects with benign serous cysts. All these intergroup differences were statistically significant. Irrespective of the group, median concentrations of sHLA-G, IL-10 and TNF-alpha in peritoneal fluid were higher than serum levels of these markers.

Conclusions: Elevated serum and peritoneal fluid concentrations of IL-10 and TNF-alpha distinguish ovarian malignancies and endometriomas from benign serous ovarian cysts. In contrast to endometriosis, ovarian malignancies are characterized by elevated peritoneal fluid concentrations of IL-10 and TNF-alpha, elevated serum concentrations of IL-10 and low serum levels of TNF-alpha. Serum and peritoneal fluid concentrations of sHLA-G have no diagnostic value in differentiating between ovarian malignancies and endometriomas.

Keywords: Ovarian cancer, Endometriosis, Ovarian cysts, IL-10, TNF-alpha, sHLA-G, Tumor escape

\footnotetext{
* Correspondence: olimpiasipak-szmigiel@wp.pl

'Department of Obstetrical and Gynecological Nursing, Pomeranian Medical

University, 48 Żołnierska, 71-210 Szczecin, Poland

Full list of author information is available at the end of the article
} 


\section{Background}

Most ovarian cancers are diagnosed in patients older than 50 years. The majority of ovarian malignancies are of epithelial origin. Histopathological type is a determinant of tumor growth, its metastatic potential, response to treatment and prognosis [1-3]. It is estimated that up to $10-15 \%$ of ovarian cancers have a genetic background [4]. Due to specific metabolic (intensive cellular metabolism, rich vascularization, local inflammation and microinjuries associated with ovulation and retrograde menstruation) and topographic features of the ovaries (location within peritoneal cavity, enabling potential malignancies to grow uncompromised and to spread locally), ovarian tumors constitute an important clinical problem. Intraperitoneal dissemination is a typical feature of ovarian cancers. Metastatic lesions are usually well-vascularized and synthesize similar mediators to those produced by the primary tumor, i.e., vascular endothelial growth factor (VEGF), tumor necrosis factor alpha (TNF-alpha), interferon alpha (INF-alpha), interleukin 2 (IL-2) and metalloproteinases; all of them play a role in the development of malignant ascites [5].

Previous studies demonstrated that $10-15 \%$ of ovarian cancer patients present with concomitant endometriosis; both conditions develop under the same environmental conditions of minor pelvis [6, 7]. One of the key processes involved in the pathogenesis of endometriosis is activation of pro-inflammatory cytokines, which is responsible for most clinical manifestations of this condition [8].

Human leukocyte antigen G (HLA-G) is a major histocompatibility complex (MHC) antigen expressed on the cell surface. A total of seven isoforms of HLA-G have been identified thus far: membrane-bound HLA-G1, HLA-G2, HLA-G3 and HLA-G4, and soluble sHLA-G5, sHLA-G6 and sHLA-G7. While the biological effects of soluble and membrane-bound HLA-G are similar, the former are observed systemically. Expression of HLA-G on the surface of cancer cells is determined by a plethora of environmental factors, including hypoxia, stress, some hormones, cytokines and viruses [9]. HLA-G can activate $T$ cells via a few various mechanisms. Interaction of HLA-G and CD4+ T cells with LILRB1 and LILRB2 receptors results in a decrease in the synthesis of T-helper 1 (Th1) cytokines, such as interferon gamma (INFgamma), IL-2 and TNF-alpha, as well as in enhanced production of Th2 cytokines, among them interleukin 3, 4 and 10 (IL-3, IL-4 and IL-10). This results in inactivation of cytotoxic $\mathrm{T}$ cells and lesser production of antitumor antibodies $[4,10]$.

IL-10 is synthesized primarily by activated $\mathrm{T}$ cells, especially Th2and $\mathrm{T}$ regulatory cells (Tregs), inter alia in response to interaction of these cells with HLA-G/ sHLA-G [11]. IL-10 is an important immunosuppressant and its elevated concentrations have been implicated in immune escape of some malignancies [12]. TNF-alpha interacts with cancer cells via its specific receptor; this results in activation of arachidonic acid cascade, enhanced generation of reactive oxygen species inside the cell and its death. However, this effect requires expression of specific receptors for TNF-alpha on the surface of cancer cells; otherwise this cytokine acts solely as an immunomodulatory agent [13].

The aim of this study was to compare serum and peritoneal fluid concentrations of sHLA-G, IL-10 and TNF-alpha in women with selected ovarian pathologies: benign serous cysts, endometrioma and malignant tumors. Moreover, we searched for correlations between serum and peritoneal fluid concentrations of these parameters. Clinical significance of using them for diagnostic purposes in women with serous ovarian cysts, endometriosis, and ovarian cancer, which in the future may improve the early diagnosis of ovarian diseases.

\section{Case Presentations}

The study involved 135 women with suspected ovarian lesions, such as cysts, endometriosis of the small pelvis, and ovarian cancer, hospitalized in the obstetrics and gynecology ward in the Independent Public Specialist Healthcare Center "Zdroje” from 2009 to 2013. The final clinical diagnosis was based on the results of histopathological examination of the material taken during a surgery, which let us distinguish three groups of women. Group 1 (G1) consisted of 54 women with benign serous ovarian cysts; the mean age was 57 years. Group 2 (G2) consisted of 43 women with endometriosis of the peritoneum, the small pelvis, and/or the ovary; the mean age was 41 years. In the group with endometriosis (G2), stage I endometriosis was not observed (n-0), stage II endometriosis was diagnosed in 35 women (n-35), and stage III-IV endometriosis - in 8 women (n-8). Group 3 (G3) comprised of 38 women with ovarian cancer; the mean age was 73 years. The patients in this group were either before $(n-31)$ or during chemotherapy (n-7). In this group, ovarian carcinomas were divided into two types: Type I $(n=12)$, and Type II $(n=26)$. In the study groups, we excluded metastatic tumors from other organs, symptoms of infection, and thyroid diseases. What is more, the women did not receive steroid agents for 3 months preceding a surgery. According to the molecular criteria of Shih and Kurman [14], epithelial ovarian cancers can be divided into two types, namely type I and type II. Endometriosis-related cancers represent type I carcinogenesis. Cancers of this type develop slowly, are histologically well-differentiated, and thus show low mitotic potential. Borderline precursor lesions can often be observed in the course of their development. Type I tumors include well-differentiated serous cancers, 
mucinous and endometrioid ovarian cancers, clear-cell carcinomas, and transitional cell cancers, which make up to $25 \%$ of all ovarian malignancies. These cancers have a relatively stable genome, and the most common mutations are found in the K-RAS, BRAF, PTEN, BCL-2, and ARIDIA genes. Type II cancers are tumors that spread rapidly, are primarily very advanced within the abdominal cavity and pelvis, and have an extremely aggressive clinical course without a previously noticeable precursor lesion. These carcinomas account for $75 \%$ of ovarian malignancies. They include poorly-differentiated serous cancers, non-differentiated cancers, and carcinosarcoma. Type II ovarian cancers are genetically unstable and are characterized by the presence of many mutations (the most frequent in the gene $p 53$ ).

The revised American Fertility Society (rAFS) has divided endometrial involvement into 4 stages or degrees, depending on its size, infiltration, the presence of cysts and adhesions. Stage I denotes minimal endometriosis, II - mild endometriosis, III - moderate endometriosis, and IV - severe endometriosis [15]. The protocol of the study was approved by the Local Bioethics Committee at the Pomeranian Medical University in Szczecin, and written informed consent was sought from all the study subjects.

Blood was taken from the patients once before operation, and peritoneal fluid was taken once intraoperatively. All laboratory tests were performed at the Assisted Reproduction Laboratory, Reproductive Medicine and Gynecology Clinic in Police. Serum and peritoneal fluid concentrations of sHLA-G were determined by means of ELISA with commercially available kits from Bio Vendor Laboratory Medicine, Inc. (catalogue no. RD194070100R). Sensitivity threshold of this assay is $3 \mathrm{U} / \mathrm{ml}$. Concentrations of IL-10 and TNF-alpha were measured by means of ELISA with specific monoclonal antibodies against these cytokines, using commercially available kits Quantikine and Quantikine HS from R\&D Systems Europe, Ltd. The sensitivity thresholds of Quantikine and Quantikine HS assays are $3.9 \mathrm{pg} / \mathrm{ml}$ and $0.03-0.17 \mathrm{pg} / \mathrm{ml}$ (mean $0.9 \mathrm{pg} / \mathrm{ml}$ ), respectively, for $\mathrm{IL}-10$, and $0.5-$ $5.5 \mathrm{pg} / \mathrm{ml}$ and $0.038-0.191 \mathrm{pg} / \mathrm{ml}$ (mean $1.6 \mathrm{pg} / \mathrm{ml}$ ), respectively, for TNF-alpha.

All statistical calculations were carried out with STATA 11 software (license no. 30110532736). Normal distributions of continuous variables were verified with Kolmogorov-Smirnov test. Statistical characteristics of quantitative variables are presented as medians, lower and upper quartiles. Depending on the distribution type and the number of analyzed groups, statistical significance of intergroup differences was verified with Student $t$-test for independent variables, Mann-Whitney $U$-test, analysis of variance (ANOVA) or Kruskal-Wallis test. The significance of intragroup differences in serum and peritoneal levels of analyzed parameters was verified with either Student $t$-test for dependent variables or Wilcoxon test. Power and direction of relationships between pairs of quantitative variables were determined on the basis of Pearson's coefficients of linear correlation or Spearman's coefficients of rank correlation. All tests were considered significant at $p \leq 0.05$.

Patients with benign serous cysts, endometrioma and ovarian malignancies did not differ significantly in terms of their serum and peritoneal fluid concentrations of sHLA-G (Table 1). Median difference between peritoneal fluid and serum concentrations of sHLA-G in ovarian cancer patients $(21.2 \mathrm{U} / \mathrm{ml})$ turned out to be significantly higher than in women with benign serous cysts $(13.04 \mathrm{U} / \mathrm{ml})$ and endometrioma $(13.00 \mathrm{U} / \mathrm{ml})$ (Fig. 1).

Ovarian cancer patients presented with significantly higher median serum concentrations of IL-10 than other study subjects. Also median concentrations of IL-10 in peritoneal fluid turned out to be the highest in ovarian cancer patients, followed by women with endometrioma and subjects with benign serous cysts. All these intergroup differences were statistically significant (Table 1 ). Irrespective of the group, median concentrations of IL-10 in peritoneal fluid were higher than serum levels of this cytokine. Median difference between peritoneal fluid and serum concentrations of IL-10 was the highest in ovarian cancer patients, followed by women with endometrioma and those with serous ovarian cysts. All these intergroup differences were statistically significant (Fig. 2).

Median serum concentrations of TNF-alpha in ovarian cancer patients were significantly higher than in other study subjects. Also median concentrations of TNFalpha in peritoneal fluid were the highest in ovarian cancer patients, followed by women with endometrioma and those with benign serous cysts. All these intergroup differences turned out to be significant on statistical analysis (Table 1). Also median difference between peritoneal fluid and serum concentrations of TNF-alpha was the highest in ovarian cancer patients, followed by women with endometrioma and serous ovarian cysts. All these intergroup differences were statistically significant (Fig. 3).

Irrespective of the group, we did not find statistically significant correlations between serum/peritoneal fluid concentrations of sHLA-G and concentrations of IL-10 and TNF-alpha in these materials. Serum concentration of IL-10 in women with benign serous cysts correlated positively with peritoneal fluid concentration of this cytokine and serum concentration of TNF-alpha; furthermore, a positive correlation between peritoneal fluid levels of IL-10 and TNF-alpha was found in this group. The only statistically significant correlation observed in endometrioma patients was a positive association 
Table 1 Serum and peritoneal fluid concentrations of sHLA-G, IL-10 and TNF-alpha in women with benign serous ovarian cysts, ovarian endometrioma and ovarian cancer (medians and interquartile ranges)

\begin{tabular}{|c|c|c|c|c|}
\hline Parameter & $\begin{array}{l}\text { Serous cysts } \\
(n=54)\end{array}$ & $\begin{array}{l}\text { Endometrioma } \\
(n=43)\end{array}$ & $\begin{array}{l}\text { Ovarian cancer } \\
(n=38)\end{array}$ & $p$ \\
\hline Serum sHLA-G (U/ml) & $8.90(5.35-21.75)$ & $7.70(5.15-17.00)$ & $10.00(5.47-14.40)$ & 0.944 \\
\hline Peritoneal fluid sHLA-G (U/ml) & $29.75(15.63-51.70)$ & $20.60(15.80-37.54)$ & 36.15 (20.68-62.08) & 0.150 \\
\hline Serum IL-10 (pg/ml) & $3.75^{*}(3.20-11.20)$ & $4.60^{*}(4.20-6.65)$ & $11.30(4.60-21.00)$ & 0.002 \\
\hline Peritoneal fluid IL-10 (pg/ml) & $18.15^{\#, \S}(4.60-41.70)$ & $53.00^{*}(14.70-93.20)$ & $128.80(36.71-252.38)$ & $<0.001$ \\
\hline Serum TNF-alpha (pg/ml) & $1.20^{\#}(1.00-1.73)$ & $1.00^{\#}(0.73-1.68)$ & $2.70(1.90-4.00)$ & $<0.001$ \\
\hline Peritoneal fluid TNF-alpha (pg/ml) & $1.80^{\#, \S}(1.00-3.80)$ & $3.10^{\ddagger}(1.70-17.50)$ & $13.21(8.35-25.35)$ & $<0.001$ \\
\hline
\end{tabular}

Significantly lower than in ovarian cancer patients: ${ }^{*} p<0.005,{ }^{\ddagger} p<0.001,{ }^{\#} p<0.0001$; significantly lower than in endometrioma patients: ${ }^{5} p<0.005$

between concentrations of IL-10 and TNF-alpha in peritoneal fluid. Serum concentration of IL-10 in ovarian cancer patients correlated positively with peritoneal fluid concentration of this cytokine and serum level of TNFalpha; moreover, women with ovarian malignancies showed a positive correlation between serum TNF-alpha an IL-10 in peritoneal fluid (Table 2). Analysis of the diagnostic sensitivity of the tested laboratory parameters in serum and peritoneal fluid. The parameters that had the highest diagnostic value, expressed as AUC >0.6, allowing for differentiating between G2 and G3 groups, were peritoneal sHLA-G concentrations, and the difference between sHLA-G concentrations in serum and peritoneal fluid; sensitivity $>70 \%$, and the level of significance $p<0.05$. The ROC graphs in Figs. 4, 5 and 6 indicate to the usefulness of peritoneal sHLA-G concentrations as a parameter differentiating between G2 and G3 groups. The cut-off peritoneal concentrations of sHLA-G $\geq 22.1 \mathrm{U} / \mathrm{ml}$, and the difference between peritoneal and serum sHLA-G concentrations $\geq 14.25 \mathrm{U} / \mathrm{ml}$ potentially indicate to ovarian cancer, while lower values suggest endometriosis. The highest differentiating value for G1 and G3 groups was attributed to peritoneal IL-10 concentrations, with a cut-off value of $34.5 \mathrm{pg} / \mathrm{ml}$, and to the difference between peritoneal and serum IL-10 concentrations, with a cut-off value of $27.6 \mathrm{pg} / \mathrm{ml}$. The most sensitive parameter $(89 \%, p<0.002)$ differentiating between G1 and G3 groups was serum IL-10 concentration, with a cut-off value of $3.8 \mathrm{pg} / \mathrm{ml}$ (Figs. 7, 8 and 9).

G1 and G3 groups, and G2 and G3 groups were best differentiated by serum and peritoneal TNF-alpha concentrations, with cut-off values of $1.55 \mathrm{pg} / \mathrm{ml}$ (G1 vs. G3) and $1.75 \mathrm{pg} / \mathrm{ml}$ (G2 vs. G3). The concentration of TNF-alpha in serum, with a cut-off value of $1.55 \mathrm{pg} / \mathrm{ml}$, was the most sensitive differentiating parameter $(89 \%$, $p<0.0001$ ) between G1 and G3 groups. G1 and G3 groups can also be differentiated by measuring serum and peritoneal TNF-alpha concentrations, with a cut-off value of $6.75 \mathrm{pg} / \mathrm{ml}$ (sensitivity of $74 \%$, specificity of $88 \%$ ) (Figs. 10, 11 and 12). Only peritoneal TNF-alpha concentrations, and the difference between peritoneal and serum TNF-alpha concentrations significantly differentiate between G1 and G2 groups.

\section{Discussion}

Ovarian malignancies represent a challenge in gynecologic oncology. This results primarily from problems with

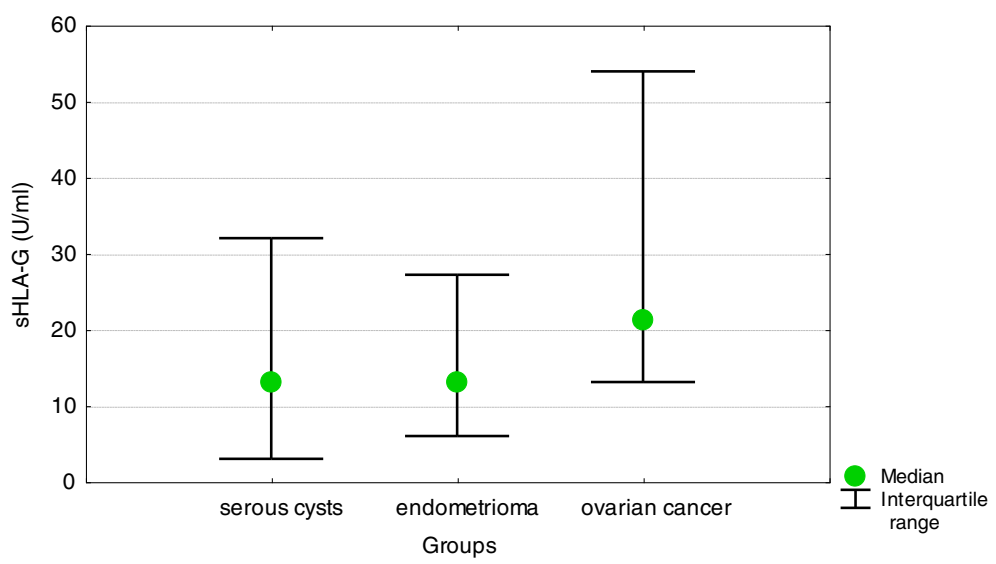

Fig. 1 Differences between peritoneal fluid and serum concentrations of sHLA-G in women with benign serous ovarian cysts, ovarian endometrioma and ovarian cancer (medians and interquartile ranges) 


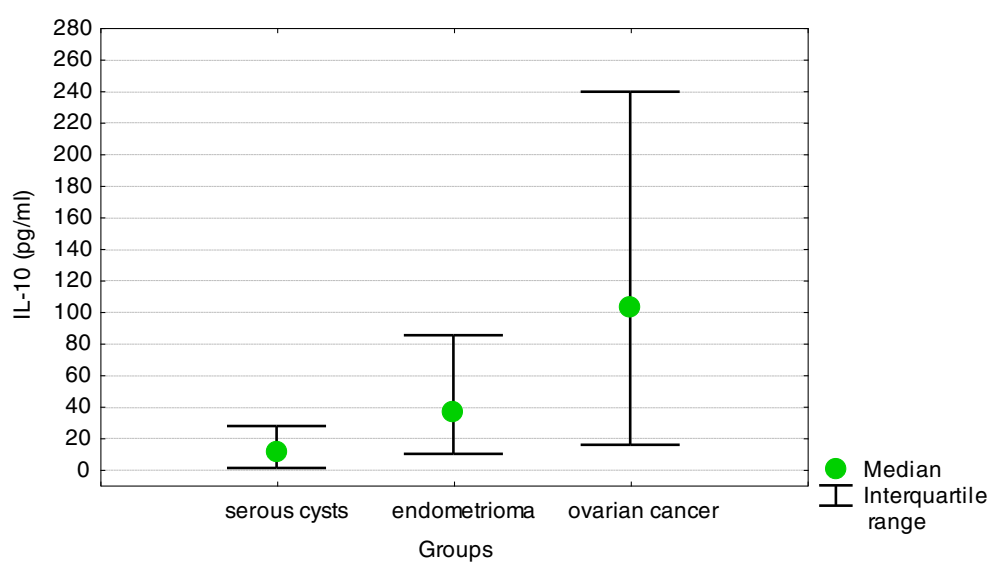

Fig. 2 Differences between peritoneal fluid and serum concentrations of IL-10 in women with benign serous ovarian cysts, ovarian endometrioma and ovarian cancer (medians and interquartile ranges)

identification of high risk groups, asymptomatic character of the disease at its early stages and lack of sufficiently specific and sensitive tests suitable for population based screening. In line with current standards, aside from clinical examination and imaging studies, evaluation of patients with suspected ovarian masses should also include determination of cancer markers [13].

Concentrations of potential cancer markers (sHLA-G, IL-10 and TNF-alpha) in body fluids (serum and peritoneal fluid) are not stable, which may be associated with both their sources and natural history of the disease. Serum concentrations of sHLA-G, IL-10 and TNF-alpha reflect systemic immune activity associated with underlying condition, whereas their levels in peritoneal fluid are associated rather with local processes.

A number of previous studies documented a link between HLA-G/sHLA-G and carcinogenesis [16-18]. Rouas-Freiss et al. [19] found expression of HLA-G on both cancer cells and tumor-infiltrating immune cells. They showed that it is the tumor itself which stimulates the expression of HLA-G/sHLA-G on both cancer cells and infiltrating immune cells, especially CD68+ macrophages and CD8+ T cells. However, they did not observe expression of these antigens in normal tissues around the tumor. Cancer patients show enhanced expression of HLA-G/sHLA-G both locally and in the blood serum. Consequently, the increase in sHLA-G concentrations in peritoneal fluid and serum of our ovarian cancer patients is consistent with previously published data [20].

Soluble HLA-G was first found in the trophoblast whereby it exerted an immunosuppressive effect, protecting fetus, recognized as a foreign antigen, against maternal immune response. Tumors and trophoblast share some common features, such as high proliferation rate, invasiveness, synthesis of growth factors and their receptors, hormones and proto-oncogenes. Furthermore, previous studies found some similarities in the immunosuppressive effects of sHLA-G expressed by pregnant women and cancer patients. According to Mach [21], expression of this antigen in cancer patients is associated

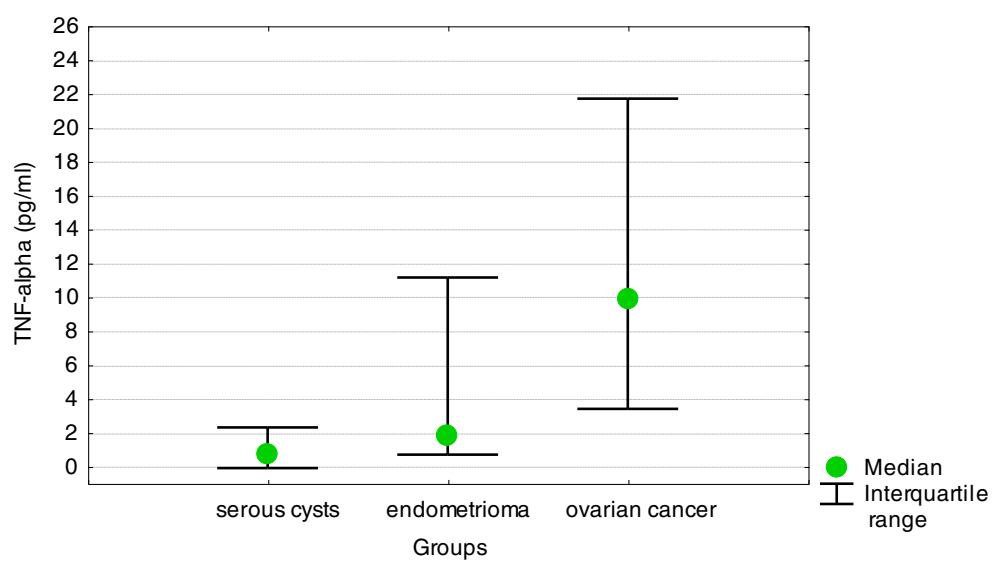

Fig. 3 Differences between peritoneal fluid and serum concentrations of TNF-alpha in women with benign serous ovarian cysts, ovarian endometrioma and ovarian cancer (medians and interquartile ranges) 
Table 2 Correlations between serum and peritoneal fluid concentrations of sHLA-G, IL-10 and TNF-alpha in women with benign serous ovarian cysts, ovarian endometrioma and ovarian cancer (Pearson's coefficients of linear correlation or Spearman's coefficients of ran correlation)

\begin{tabular}{|c|c|c|c|c|c|}
\hline Variable & $\begin{array}{l}\text { Peritoneal } \\
\text { SHLA-G }\end{array}$ & $\begin{array}{l}\text { Serum } \\
\text { IL-10 }\end{array}$ & $\begin{array}{l}\text { Peritoneal } \\
\text { IL-10 }\end{array}$ & $\begin{array}{l}\text { Serum } \\
\text { TNF-alpha }\end{array}$ & $\begin{array}{l}\text { Peritoneal } \\
\text { TNF-alpha }\end{array}$ \\
\hline \multicolumn{6}{|l|}{ Serous cysts $(n=54)$} \\
\hline Serum sHLA-G & $0.07(p=0.635)$ & $-0.19(p=0.178)$ & $-0.07(p=0.637)$ & $0.13(p=0.371)$ & $-0.23(p=0.116)$ \\
\hline Peritoneal sHLA-G & & $0.13(p=0.362)$ & $0.16(p=0.253)$ & $-0.10(p=0.483)$ & $0.06(p=0.662)$ \\
\hline Serum IL-10 & & & $0.42(p=0.002)$ & $0.35(p=0.013)$ & $0.14(p=0.322)$ \\
\hline Peritoneal IL-10 & & & & $0.09(p=0.527)$ & $0.42(p=0.002)$ \\
\hline Serum TNF-alpha & & & & & $0.22(p=0.133)$ \\
\hline \multicolumn{6}{|c|}{ Endometrioma $(n=43)$} \\
\hline Serum sHLA-G & $0.15(p=0.348)$ & $-0.06(p=0.715)$ & $-0.06(p=0.724)$ & $-0.04(p=0.800)$ & $-0.06(p=0.714)$ \\
\hline Peritoneal sHLA-G & & $0.05(p=0.754)$ & $0.10(p=0.517)$ & $0.26(p=0.110)$ & $-0.02(p=0.875)$ \\
\hline Serum IL-10 & & & $0.15(p=0.350)$ & $0.11(p=0.495)$ & $-0.16(p=0.318)$ \\
\hline Peritoneal IL-10 & & & & $0.09(p=0.581)$ & $0.50(p=0.001)$ \\
\hline Serum TNF-alpha & & & & & $0.25(p=0.123)$ \\
\hline \multicolumn{6}{|c|}{ Ovarian cancer $(n=38)$} \\
\hline Serum sHLA-G & $0.15(p=0.389)$ & $0.04(p=0.806)$ & $-0.19(p=0.270)$ & $0.22(p=0.201)$ & $-0.03(p=0.860)$ \\
\hline Peritoneal sHLA-G & & $-0.17(p=0.328)$ & $-0.11(p=0.500)$ & $-0.12(p=0.511)$ & $0.21(p=0.205)$ \\
\hline Serum IL-10 & & & $0.50(p=0.003)$ & $0.50(p=0.003)$ & $-0.12(p=0.501)$ \\
\hline Peritoneal IL-10 & & & & $0.40(p=0.021)$ & $0.27(p=0.106)$ \\
\hline Serum TNF-alpha & & & & & $0.27(p=0.126)$ \\
\hline
\end{tabular}

with progression of the malignancy and worse prognosis. Basta et al. [22] analyzed the role of HLA-G in progression of recurrent ovarian cancer. They found expression of this antigen both on cancer cells and on macrophages infiltrating the tumor and its microenvironment. The study conducted by these authors included two groups of women with recurrent ovarian cancer, with complete remission after primary treatment and without. The latter group presented with higher concentrations of HLA-G within the tumor, which implies that expression of this antigen may be associated with cancer stage.

Our findings are also consistent with the results presented by Matalliotakis et al. [23], according to whom women with active endometriosis present with lower

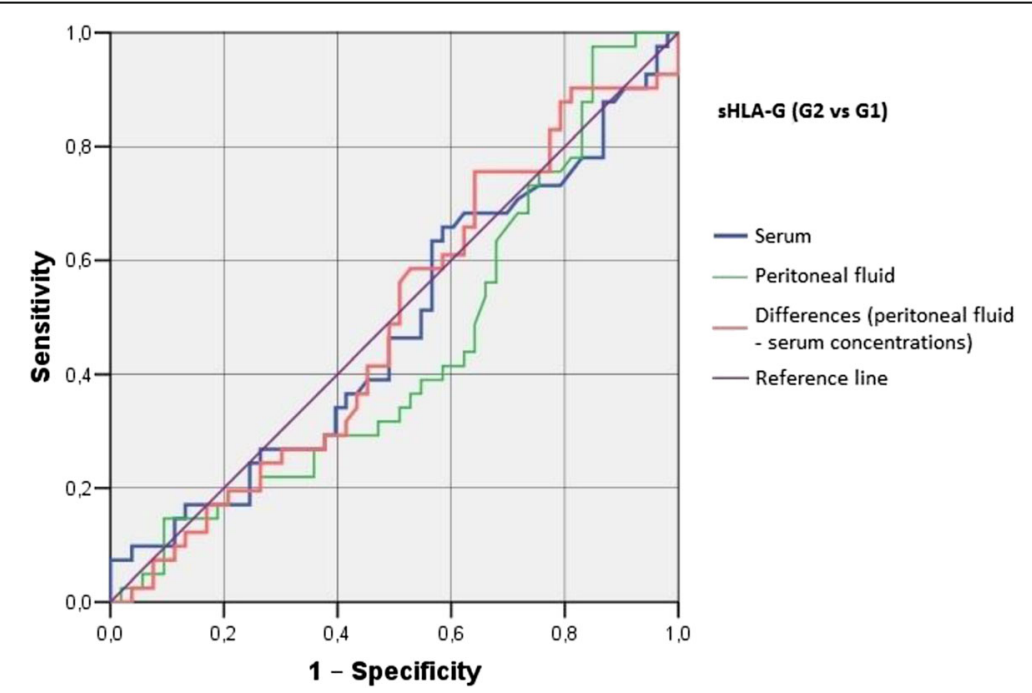

Fig. 4 The ROC graph showing serum and peritoneal SHLA-G concentrations, and the difference in peritoneal and serum concentrations between G2 and G1 groups 


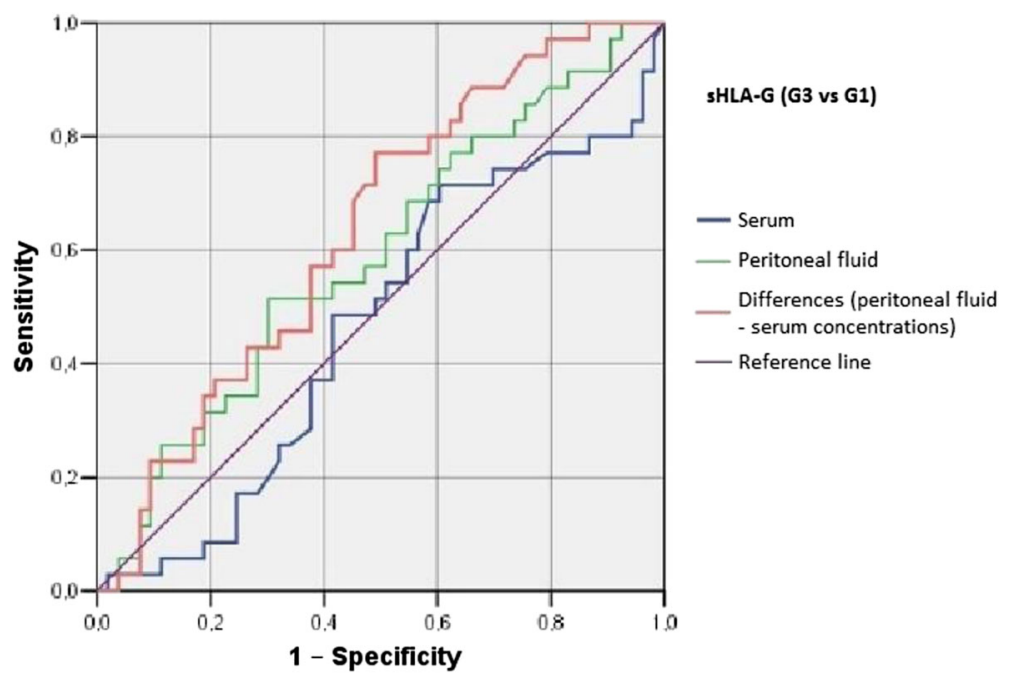

Fig. 5 The ROC graph showing serum and peritoneal SHLA-G concentrations, and the difference in peritoneal and serum concentrations between G3 and G1 groups

serum levels of sHLA-G. The same study showed that serum level of sHLA-G normalizes after treatment and then decreases again whenever the symptoms of endometriosis recur. Our results are also in line with those reported by Eidukaite and Tamosiunas [24], who found that concentrations of sHLA-G in peritoneal fluid from women with endometriosis and without are similar. Barrier et al. [25] demonstrated that whereas HLA-G is expressed by endometroid cells, it is absent in normal endometrial cells. However, Wang et al. [26] found the expression of HLA-G in both normal and ectopic endometrium, and Hornung et al. [27] did not detect this antigen in either normal endometrium or endometrial foci. All these discrepancies may reflect differences in the analyzed material and examined parameters (HLA-G vs. sHLA-G).

In our study, women with serous ovarian cysts showed a considerable variability in both serum and peritoneal fluid concentrations of sHLA-G, and no significant intergroup differences were found with regards to these parameters. To the best of our knowledge, there is no published evidence documenting a link between sHLA$\mathrm{G}$ concentration and serous ovarian cysts. However, it should be remembered that concentration of sHLA-G in patients with various conditions may change across their menstrual cycle; this might affect our findings and contribute to a relatively low discriminatory power of this parameter. In line with the surgical protocol, material

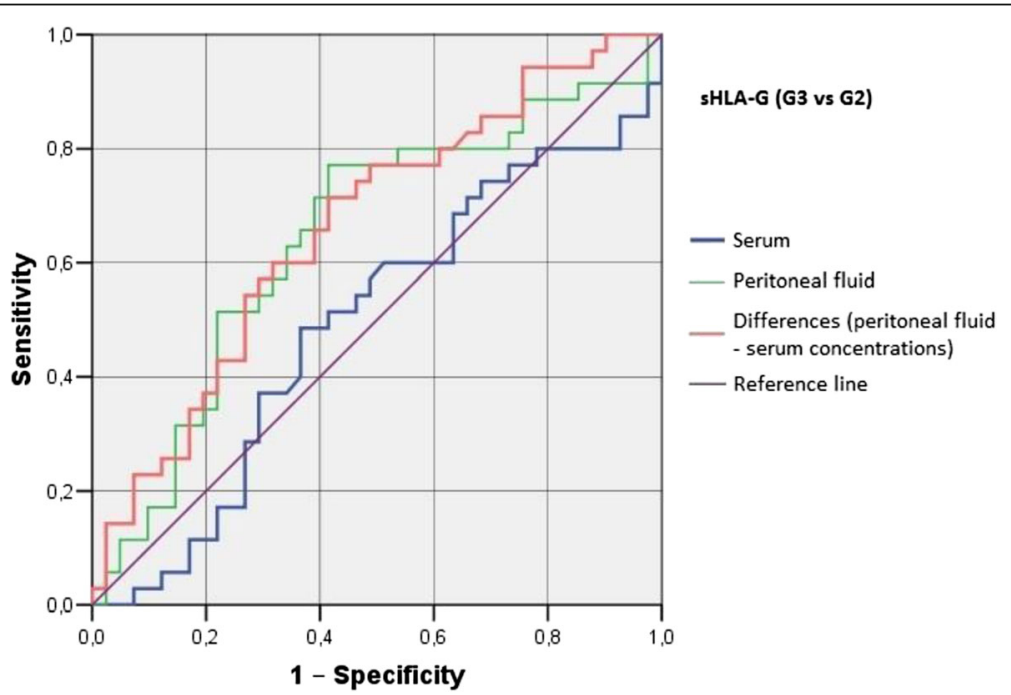

Fig. 6 The ROC graph showing serum and peritoneal SHLA-G concentrations, and the difference in peritoneal and serum concentrations between G3 and G2 groups 


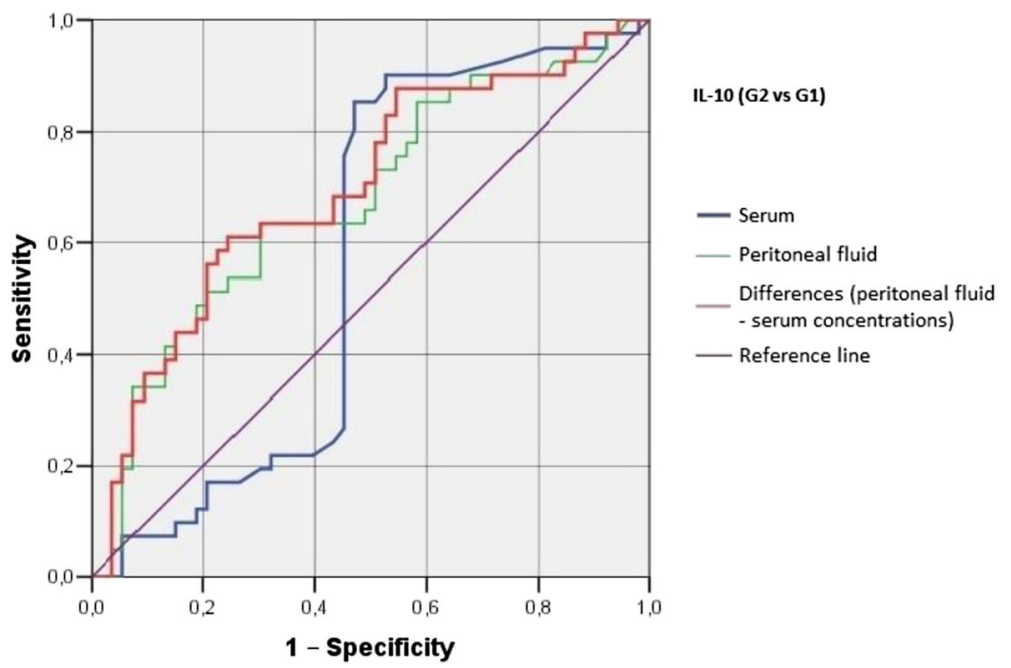

Fig. 7 The ROC graph showing serum and peritoneal IL-10 concentrations, and the difference in peritoneal and serum concentrations between G2 and G1 groups

from women with endometriosis was collected during the $1^{\text {st }}$ phase of menstrual cycle. In turn, the time of sampling in patients with serous ovarian cysts was highly variable and some of these women were amenorrheic. Mach et al. [28] analyzed phase-specific changes in serum concentrations of sHLA-G in patients with endometriosis and ovarian carcinomas. They found that irrespective of the group, serum concentrations of sHLA-G were always higher during the $1^{\text {st }}$ phase of the cycle. However, while the levels of sHLA-G in patients with ovarian cancer and deep endometriosis remained elevated during further stages of the cycle as well, a significant decrease in this parameter was observed during the secretory phase in women with ovarian endometrioma. This evidence points to the $1^{\text {st }}$ phase of menstrual cycle as an optimal timing to determine serum concentration of sHLA-G.

Clinical importance of HLA-G/sHLA-G is a subject of an extensive research, as shown by a plethora of recently published papers documenting biological effects of these antigens. Sheu and Shih [17] described the mechanisms via which sHLA-G interacts with immune cells. Dimerics HLA-G impairs the ability of dendritic cells to present antigen, activates suppressor lymphocytes and inhibits synthesis of interleukin 12 (IL-12). In turn, sHLA-G monomer is responsible for functional inhibition of $\mathrm{B}$ cells due to induction of their apoptosis and promotion of Th2 cytokine (IL-10, IL-3 and IL-4) production. Due to their immunosuppressive character, these cytokines impair

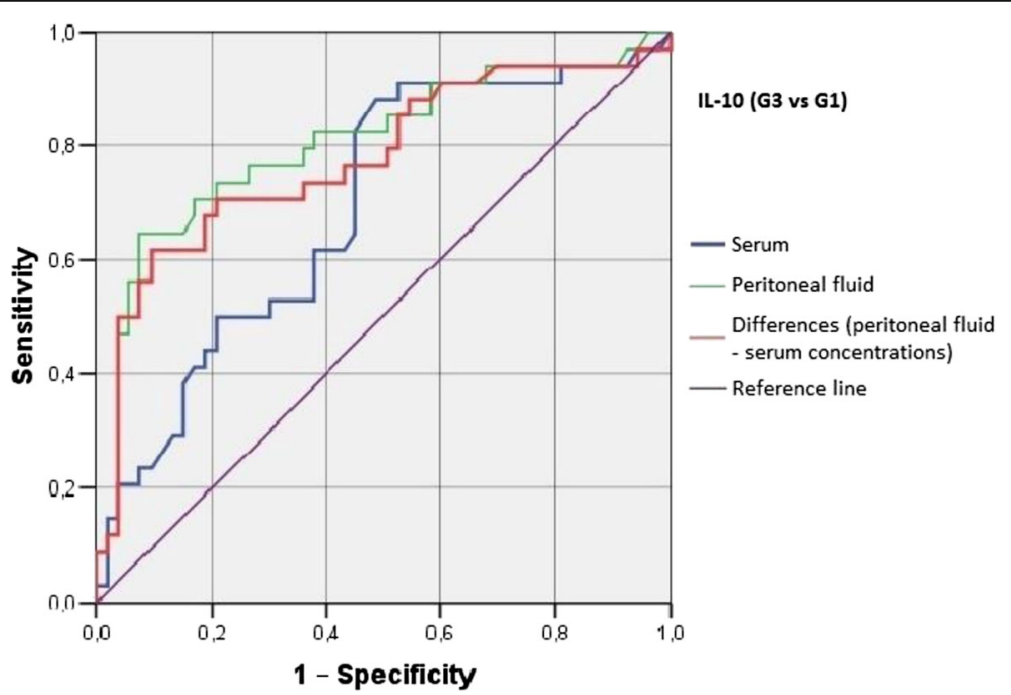

Fig. 8 The ROC graph showing serum and peritoneal IL-10 concentrations, and the difference in peritoneal and serum concentrations between G3 and G1 groups 


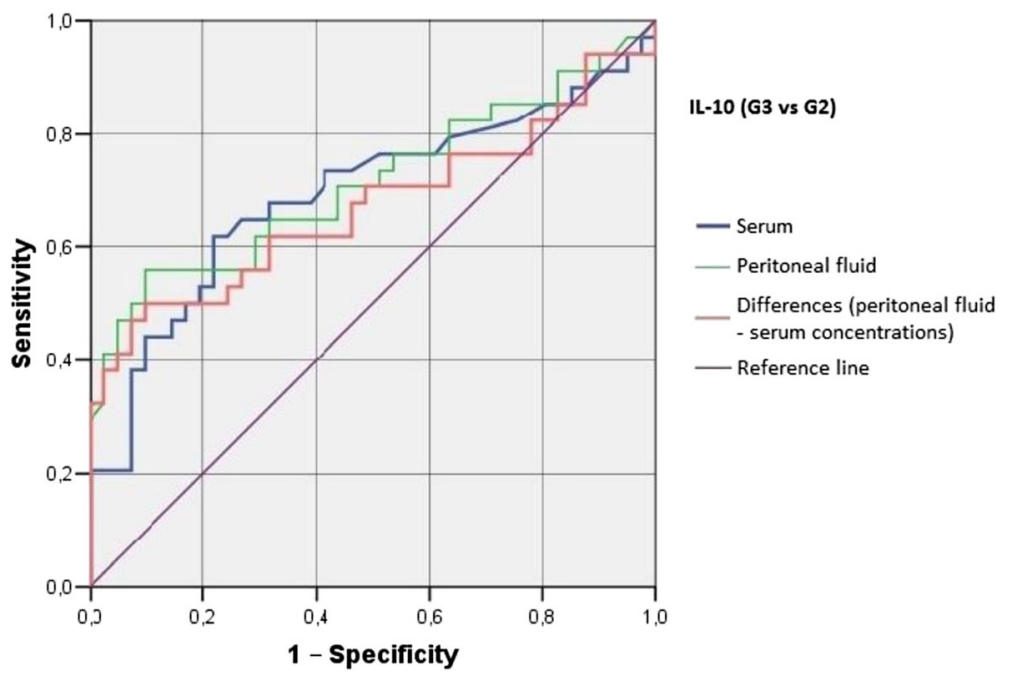

Fig. 9 The ROC graph showing serum and peritoneal IL-10 concentrations, and the difference in peritoneal and serum concentrations between G3 and $G 2$ groups

anti-inflammatory and anti-tumor response. The same authors [18] found expression of HLA-G on ovarian cancer cells and documented its role in immune escape of this malignancy.

We did not find significant differences in serum concentrations of sHLA-G in patients with benign serous ovarian cysts, endometrioma and ovarian tumors. Based on available evidence we expected that patients with endometrioma and ovarian malignancies will present with lower and higher levels of sHLA-G, respectively. However, this hypothesis was not confirmed, probably due to a substantial variability of individuals results in both groups. Also the difference in serum concentrations of sHLA-G in patients with endometriomas and serous ovarian cysts was not statistically significant. A number of factors may explain the lack of significant intergroup differences. First, as already mentioned, HLA-G is present in both membrane-bound and soluble form. Although both of them exert similar biological effects, it should be remembered that our study was limited solely to sHLA-G. Another potential explanation for the lack of higher concentrations of sHLA-G in ovarian cancer patients may be the fact that this antigen is expressed only by some ovarian cancer lines. Singer et al. [20] examined sHLA-G as a potential marker of ascites; they showed that although the sensitivity of this parameter in the differential diagnosis of malignant and benign ascites is similar as that of cytological examination, contrary to

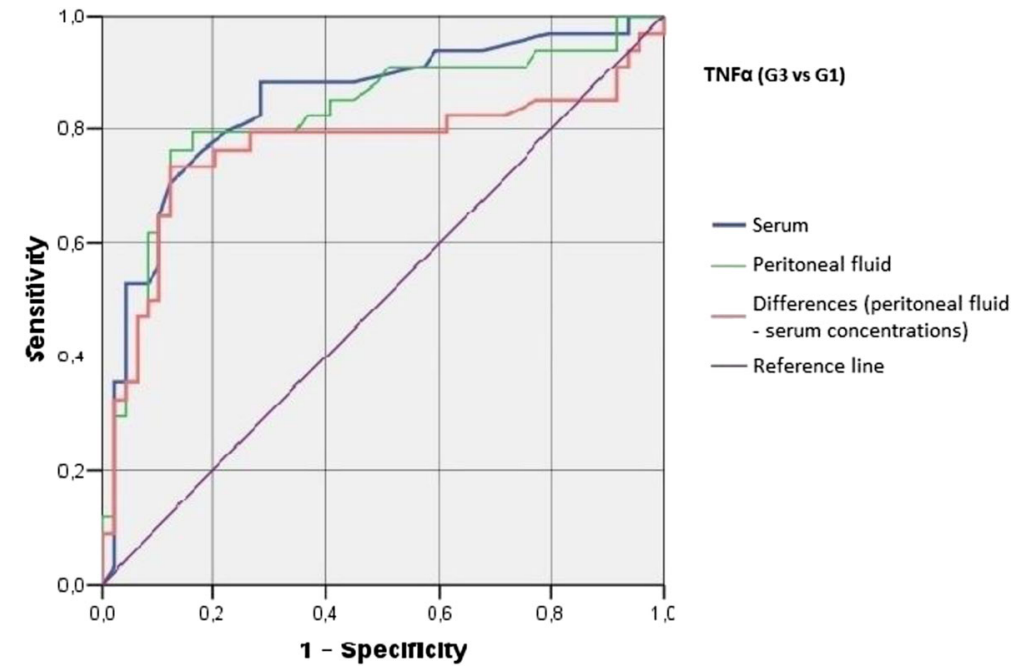

Fig. 10 The ROC graph showing serum and peritoneal TNF-alpha concentrations, and the difference in peritoneal and serum concentrations between $\mathrm{G} 2$ and $\mathrm{G} 1$ groups 


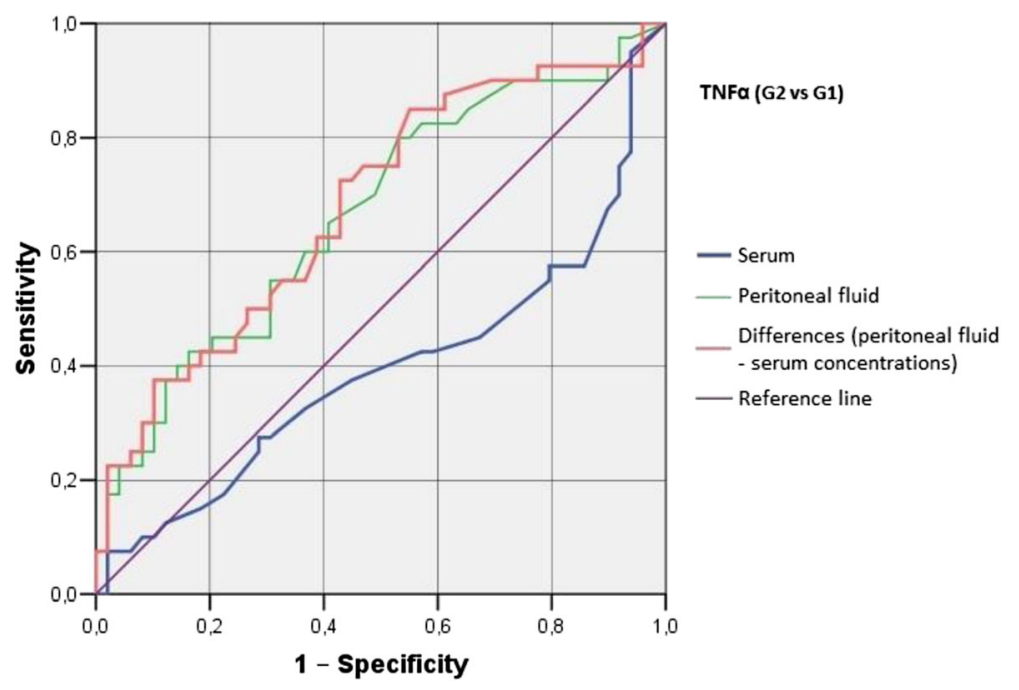

Fig. 11 The ROC graph showing serum and peritoneal TNF-alpha concentrations, and the difference in peritoneal and serum concentrations between $\mathrm{G} 3$ and $\mathrm{G} 1$ groups

the former it cannot be used to establish an ultimate diagnosis. Consequently, determination of sHLA-G seems to be primarily applicableto research on the pathomechanisms of ovarian cancer and endometriosis.

Presence of tumor is associated with local inflammation resulting from infiltration with immune cells, autocrine and paracrine release of cytokines. Moradi et al. [29] analyzed concentrations of TNF-alpha, interleukin 1 and 6(IL-1and IL-6) in serum and peritoneal fluid from healthy women and ovarian cancer patients, and demonstrated that presence of malignancy was associated with a significant increase in all these parameters. The increase in TNF-alpha level results from activation of immune response to ovarian tumor. The synthesis of this cytokine depends on tumor-specific cells, primarily tumorassociated macrophages. Kulbe et al. [30] found elevated concentrations of pro-inflammatory cytokines, such as TNF-alpha, C-X-C motif chemokine ligand 12 (CXCL12) and IL-6 in many cancer cell lines, and documented their role in angiogenesis and infiltration. Our findings are consistent with observations of these authors, pointing to a link between TNF-alpha and carcinogenesis. Complex role of TNF-alpha in tumor pathogenesis was a subject of many studies; their authors tried to explain why this proinflammatory cytokine either contributes to cancer control or promotes its growth and spread.

Endometriosis is characterized by chronic inflammation resulting from synthesis of many pro-inflammatory and

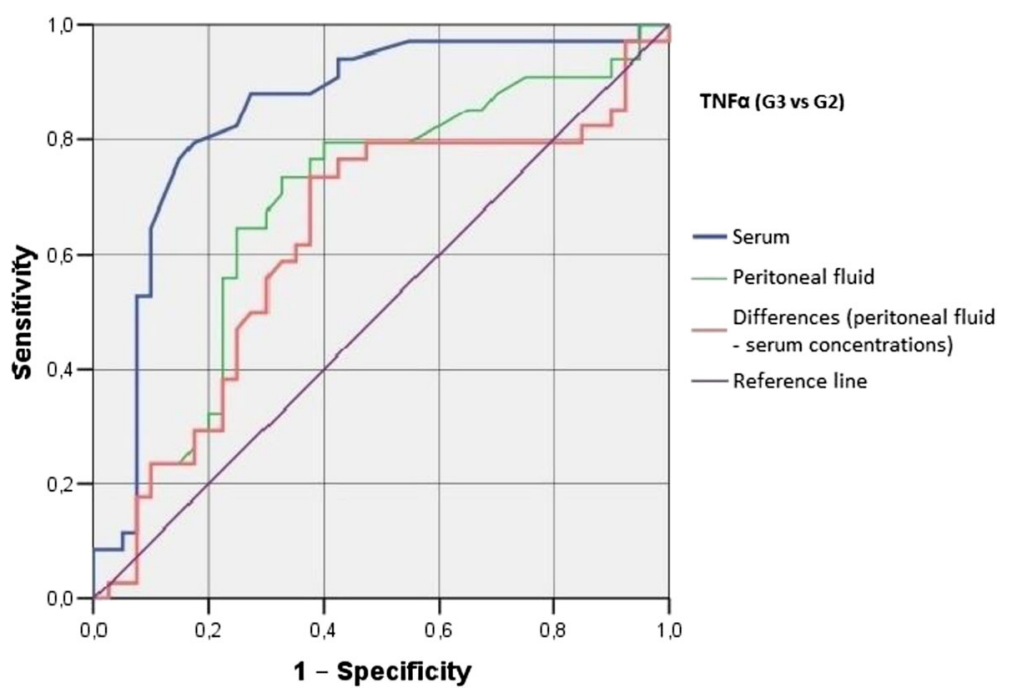

Fig. 12 The ROC graph showing serum and peritoneal TNF-alpha concentrations, and the difference in peritoneal and serum concentrations between $\mathrm{G} 3$ and $\mathrm{G} 2$ groups 
immunosuppressive cytokines. Interactions between various cytokines, including TNF-alpha, are responsible for aggravation of inflammatory processes, resultant hyperemia, pain, tissue injury, formation of adhesions and dysfunction of various organs and systems. One of the consequences is impaired fertility. Koninckxet al. [31] analyzed the role of TNF-alpha in pelvic inflammation and pain associated with endometriosis, and verified if these ailments may be attenuated with anti-TNF monoclonal antibodies.

Our observation that women with endometriomas present with higher peritoneal fluid concentrations of TNF-alpha is consistent with the results of previous studies conducted by Eisermann et al. [32], Braun et al. [33] and Rana et al. [34]. Also according to Podgaec et al. [35], women with endometriosis present with elevated peritoneal fluid concentrations of TNF-alpha and other Th1 cytokines, as well as which higher levels of IL-10 and other Th2 cytokines. In turn, Xavier et al. [36] demonstrated that irrespective of the cycle phase, endometriosis is associated with elevated serum concentrations of TNF-alpha. According to Zhang et al. [37], the increase in TNF-alpha level promotes adhesion of endometrial cells to peritoneum both in vitro and in vivoand therefore, may play a role in the pathogenesis of endometriosis. Similar to our study, also other authors reported elevated serum concentrations of TNF-alpha in women with endometriosis and ovarian cancer [37-39]. Bedaiwy et al. [38] compared diagnostic value of CA125 and other serum, peritoneal fluid, tissue and genetic markers of endometriosis; the only parameters suitable for differential diagnosis of this condition were concentration of TNF-alpha in peritoneal fluid and serum level of IL-6. In turn, Galo et al. [40] demonstrated that women with endometriosis present with elevated serum levels of TNF-alpha and in contrary to our study, identified this parameter as an accurate diagnostic marker of this condition.

According to Zhou et al. [41], peritoneal fluid concentration of IL-10 is significantly higher than serum concentration of this cytokine. The same authors demonstrated that ovarian cancer patients present with significantly higher values of this parameter than women with benign lesions, which is also consistent with our findings. Furthermore, they observed that concentration of IL-10 in ovarian cancer cell lysate was markedly higher than its serum level, which points to tumor microenvironment as a principal source of this cytokine. According to Berger et al. [42], IL-10 can be synthesized by various ovarian cancer cell lines. Berek et al. [43] documented lower cytotoxicity of lymphocytes isolated from serum and peritoneal fluid of ovarian cancer patients. One potential explanation for this phenomenon was provided by Rouas-Freiss et al. [19]; similar to our study, these authors demonstrated that ovarian cancer patients present with elevated serum levels of IL-10 and other immunomodulatory cytokines, such as leukemia inhibitory factor (LIF), granulocyte macrophage colony stimulating factor (GM-CSF) and IFN-gamma.

Similar to our study, Mustea et al. [44] demonstrated that ovarian cancer patients, especially those with serous tumors, present with elevated peritoneal fluid concentrations of IL-10, markedly higher than serum levels of this cytokine. Higher concentrations of IL-10 were associated with more advanced clinical stages of ovarian cancer, which was also reported by Cho and Shih [2]. Elevated concentrations of IL-10 in ascites fluid from ovarian cancer patients were also reported by Yigit et al. [45] and Matte et al. [46]. These findings imply that due to its immunosuppressive effects, IL-10 likely interferes with both systemic and local anti-tumor response and promotes progression of ovarian malignancies.

Mutual interactions between cytokines, their influence on the cells of the immune system and the expression of surface antigens, as well as multifactor regulation of their secretion cause that sHLA-G, IL-10 and TNF-alpha are functionally related and their concentrations can correlate with each other. Cho [2] as well as Sheu and Shih [18] found that high expression of HLA-G/sHLA-G and a high level of IL-10 in the micro-environment of ovarian cancer contribute to an aggressive course of the disease. They explained the mechanisms of mutual interactions between various forms of HLA-G and immune system cells. Bukur et al. [47] described the molecular basis of HLA-G expression in various neoplasms, and immunosuppressive interactions between sHLA-G and IL-10 in kidney cancer.

Yoon et al. [48] analyzed the expression of HLA-G and its impact on the IL-10 concentration in cervical cancer, and reported on a relationship between the produced IL-10 and sHLA-G expression.

A similar relationship may also exist between TNFalpha and IL-10, as they represent two opposite but complementary types of immunological response. After primary activation of proinflammatory processes, characterized by the presence of TNF-alpha, the inflammatory response is suppressed through the activation of immunosuppressive mechanisms and the release of IL10. In chronic inflammatory states, these cytokines exist next to each other, competing in the micro-environment in a peculiar way.

Cassatella et al. [49] studied inhibiting effects of IL-10 on the production of TNF $\alpha$ through neutrophils stimulated with lipopolysaccharide, and noticed that the production of TNF-alpha was inhibited proportionally to an increase in IL-10 concentration, which suggests that there is a negative correlation between TNF-alpha and IL-10. Kanai et al. [50] demonstrated that soluble HLA- 
G stimulates the release of TNF-alpha and INFY by peripheral blood mononuclear cells, and reduces IL-3 secretion, suggesting that sHLA-G and TNF-alpha are correlated, which, however, was not confirmed by our findings. Summing up, our study shows that in most cases, concentrations of the tested indicators are not related to each other. Hence a diagnostic justification for measuring each of them.

Kleinberg et al. [51] studied the relationship of HLA-G to breast cancer and pleural mesothelioma. When analyzing the tumor and effusion fluid in its area, they found that the prognostic value of sHLA-G expression can differ depending on the type of neoplasm.

Similarly, Ye et al. [52] investigated HLA-G expression and assessed its prognostic value in patients with colorectal cancer, finding it to be an independent prognostic factor in these patients.

Sebti et al. [53] and Gros et al. [54], who gauged immunological significance of sHLA-G in patients with chronic lymphocytic leukemia, obtained comparable results.

In our study, only slight differences in serum sHLA-G levels were observed between the groups of women. It is true that sHLA-G levels were low in the group with endometriosis and high in the group with ovarian cancer. Nevertheless, discrepancies in SHLA-G levels within each of these groups caused that they did not differentiate ovarian cancer from non-cancerous lesions. The difference in sHLA-G levels between the group with endometriosis and the group with serous cysts was not statistically significant. In the organism, HLA-G can occur in membrane-bound and soluble forms, both of them having a similar biological effect. In our study, only the soluble form of HLA-G was taken into account. sHLA-G is only present in some ovarian cancer cell lines, therefore in many cases of this disease its concentration is low.

Singer et al. [20] made an attempt at using sHLA-G as a tumor marker to diagnose ascites, and achieved sensitivity comparable with cervical cytology. However, unlike cervical cytology, the use of sHLA-G gave no final, unambiguous diagnosis.

It seems that measuring sHLA-G levels is mainly of cognitive value in the analysis of the pathomechanisms involved in the development of ovarian cancer and endometriosis.

Bedaiwy et al. [38] compared the use of Ca125 in detecting endometriosis with various serum, peritoneal fluid, tissue, and genetic markers, and concluded that only TNF-alpha in peritoneal fluid and IL-6 in serum were of significant diagnostic value.

Likewise, Galo et al. [40] observed that women with endometriosis had increased serum TNF-alpha levels. In their prospective clinical research, these authors found that TNF-alpha in serum was a good marker for endometriosis, which was not supported by our findings, showing no significant differences between the groups.

\section{Conclusions}

Elevated serum and peritoneal fluid concentrations of IL-10 and TNF-alpha distinguish ovarian malignancies and endometriomas from benign serous ovarian cysts. In contrast to endometriosis, ovarian malignancies are characterized by elevated peritoneal fluid concentrations of IL-10 and TNF-alpha, elevated serum concentrations of IL-10 and low serum levels of TNF-alpha. Serum and peritoneal fluid concentrations of sHLA-G have no diagnostic value in differentiating between ovarian malignancies and endometriomas.

\section{Abbreviations}

ANOVA: Analysis of variance; CXCL12: C-X-C motif chemokine ligand 12; GM-CSF: Granulocyte macrophage colony stimulating factor (GM-CSF); HLA-G: Human leukocyte antigen; IFN-alpha: Interferon alpha; IFNgamma: Interferon gamma; IL-10: Interleukin 10; IL-12: Interleukin 12; IL2: Interleukin 2; IL-3: Interleukin 3; IL-4: Interleukin 4; LIF: Leukemia inhibitory factor; MHC: Major histocompatibility complex; sHLA-G: Soluble human leukocyte antigen; Th cells: T-helper cells; TNF-alpha: Tumor necrosis factor alpha; Tregs: T-regulatory cells; VEGF: Vascular endothelial growth factor

Funding

This study received no special funding.

\section{Availability of data and materials}

The dataset supporting the conclusions of this article is included within the article and its additional files.

\section{Authors' contributions}

OSS: Experimental design, data analysis and interpretation, manuscript composition. PW: Experimental design, data analysis and interpretation, manuscript edition. ERW: Data analysis and interpretation, manuscript edition. AN: Experimental design. BK: Data analysis and helped in writing the manuscript. SSG: Data analysis and helped in writing the manuscript. ML: Supervised the work, manuscript edition and corrected the final version of the manuscript. WM: Manuscript edition. All authors read and approved the final manuscript.

\section{Competing interests}

The authors declare that they have no competing interests.

Consent for publication

Not applicable.

\section{Ethics approval and consent to participate}

The protocol of the study was approved by the Local Bioethics Committee at the Pomeranian Medical University in Szczecin, and written informed consent was sought from all the study subjects.

\section{Publisher's Note}

Springer Nature remains neutral with regard to jurisdictional claims in published maps and institutional affiliations.

\section{Author details}

${ }^{1}$ Department of Obstetrical and Gynecological Nursing, Pomeranian Medical University, 48 Żołnierska, 71-210 Szczecin, Poland. ${ }^{2}$ Clinical Hospital SPS ZOZ "Zdroje", Mączna 4, 70-780 Szczecin, Poland. ${ }^{3}$ Clinic of Maternofetal Medicine and Gynecology, Pomeranian Medical University in Szczecin, Unii Lubelskiej 1, 71-242 Szczecin, Poland. ${ }^{4}$ Public Health Department, Pomeranian Medical University in Szczecin, Żołnierska 48, 71-210 Szczecin, Poland. ${ }^{5}$ Deparment of 
Physiology, Pomeranian Medical University in Szczecin, al. Powstańców Wlkp. 72, 70-111 Szczecin, Poland. ${ }^{6}$ Department of Histology and Developmental Biology, Pomeranian Medical University in Szczecin, Żołnierska 48, 71-210 Szczecin, Poland

\section{Received: 21 November 2016 Accepted: 22 March 2017}

\section{Published online: 04 April 2017}

\section{References}

1. Barrier BF. Immunology of endometriosis. Clin Obstet Gynecol. 2010;53:397402.

2. Cho KR, Shih IM. Ovarian cancer. Annu Rev Pathol. 2009;4:287-313.

3. Ulukus $M$, Arici A. Immunology of endometriosis. Minerva Ginecol. 2005;57:237-48

4. Chung EY, Liu J, Homma Y, Zhang Y, Brendolan A, Saggese M, et al. Interleukin-10 expression in macrophages during phagocytosis of apoptotic cells is mediated by homeodomain proteins Pbx1 and Prep-1. Immunity. 2007:27:952-64

5. Sangisetty SL, Miner TJ. Malignant ascites: a review of prognostic factors, pathophysiology and therapeutic measures. World J Gastrointest Surg. 2012:4:87-95.

6. Aris A. Endometriosis-associated ovarian cancer: a ten-year cohort study of women living in the Estrie Region of Quebec, Canada. J Ovarian Res. 2010:3:1757-2215.

7. Worley MJ, Welch WR, Berkowitz RS, Ng SW. Endometriosis-associated ovarian cancer: a review of pathogenesis. Int J Mol Sci. 2013;14:5367-79.

8. Lee KS, Baek DW, Kim KH, Shin BS, Lee DH, Kim JW, et al. IL-10-dependent down-regulation of $\mathrm{MHC}$ class II expression level on monocytes by peritoneal fluid from endometriosis patients. Int Immunopharmacol. 2005:5:1699-712.

9. Hviid TV, Hylenius S, Hoegh AM, Kruse C, Christiansen OB. HLA-G polymorphisms in couples with recurrent spontaneous abortions. Tissue Antigens. 2002;60:122-32.

10. Kobayashi H, Higashiura $Y$, Shigetomi H, Kajihara H. Pathogenesis of endometriosis: the role of initial infection and subsequent sterile inflammation (Review). Mol Med Rep. 2014;9:9-15.

11. Zheng XQ, Li CC, Xu DP, Lin A, Bao WG, Yang GS, et al. Analysis of the plasma soluble human leukocyte antigen-G and interleukin-10 levels in childhood atopic asthma. Hum Immunol. 2010;71:982-7.

12. Maccio A, Madeddu C. Inflammation and ovarian cancer. Cytokine. 2012;58:133-47.

13. Knutson KL, Karyampudi L, Lamichhane P, Preston C. Targeted immune therapy of ovarian cancer. Cancer Metastasis Rev. 2015;34:53-74

14. Shih I, Kurman R. Ovarian tumor genesis: a proposed model based on morphological and molecular genetic analysis. Am J Pathol. 2004;164:1511-8.

15. Canis M, Donnez J, Guzick D, Halme JRock J, Schenken R, et al. Revised American Society for Reproductive Medicine classification of endometriosis: 1996. Fertil Steril. 1997;67:817-21.

16. Sayed D, Badr G, Maximous D, Mikhail NN, Abu-Tarboush F, Alhazza IM. HLA-G and its relation to proliferation index in detection and monitoring breast cancer patients. Tissue Antigens. 2010;75:40-7.

17. Sheu J, Shih IM. HLA-G and immune evasion in cancer cells. J Formos Med Assoc. 2010;109:248-57.

18. Sheu JJ, Shih IM. Clinical and biological significance of HLA-G expression in ovarian cancer. Semin Cancer Biol. 2007;17:436-43.

19. Rouas-Freiss N, Moreau P, Ferrone S, Carosella ED. HLA-G proteins in cancer: do they provide tumor cells with an escape mechanism? Cancer Res. 2005;65:10139-44.

20. Singer G, Rebmann V, Chen YC, Liu HT, Ali SZ, Reinsberg J, et al. HLA-G is a potential tumor marker in malignant ascites. Clin Cancer Res. 2003:9:4460-4.

21. Mach P. The clinical significance of HLA-G in the context of gynecological cancer and conditions associated with pregnancy. Arch Perinatal Med. 2013:19:156-65

22. Basta P, Gałązka K, Stasienko E, Dutsch-Wicherek M, Wicherek $Ł$. Analysis of B7H4 and HLA-G immunoreactivity within ovarian cancer relapse and its microenvironment according to the preceding applied chemotherapy. Curr Gynecol Oncol. 2011;9:9-17.

23. Matalliotakis IM, Athanassakis I, Goumenou AG, Neonaki MA, Koumantakis EE, Vassiliadis $\mathrm{S}$, et al. The possible anti-inflammatory role of circulating human leukocyte antigen levels in women with endometriosis after treatment with danazol and leuprorelin acetate depot. Mediators Inflamm. 2001;10:75-80

24. Eidukaite A, Tamosiunas V. Soluble HLA-G in the peritoneal fluid of women with endometriosis. Fertil Steril. 2008:89:465-7.

25. Barrier BF, Kendall BS, Ryan CE, Sharpe-Timms KL. HLA-G is expressed by the glandular epithelium of peritoneal endometriosis but not in eutopic endometrium. Hum Reprod. 2006;21:864-9.

26. Wang F, Wen Z, Li H, Yang Z, Zhao X, Yao X. Human leukocyte antigen-G is expressed by the eutopic and ectopic endometrium of adenomyosis. Fertil Steril. 2008;90:1599-604.

27. Hornung D, Fujii E, Lim KH, Vigne JL, McMaster MT, Taylor RN. Histocompatibility leukocyte antigen-G is not expressed by endometriosis or endometrial tissue. Fertil Steril. 2001:75:814-7.

28. Mach P, Blecharz P, Basta P, Marianowski P, Skret-Magierlo J, Kojs Z, et al. Differences in the soluble HLA-G blood serum concentration levels in patients with ovarian cancer and ovarian and deep endometriosis. Am J Reprod Immunol. 2010;63:387-95.

29. Moradi MM, Carson LF, Weinberg B, Haney AF, Twiggs LB, Ramakrishnan S Serum and ascitic fluid levels of interleukin-1, interleukin-6, and tumor necrosis factor-alpha in patients with ovarian epithelial cancer. Cancer. 1993:72:2433-40.

30. Kulbe H, Chakravarty P, Leinster DA, Charles KA, Kwong J, Thompson RG, et al. A dynamic inflammatory cytokine network in the human ovarian cancer microenvironment. Cancer Res. 2012;72:66-75.

31. Koninckx PR, Craessaerts M, Timmerman D, Cornillie F, Kennedy S. Anti-TNFalpha treatment for deep endometriosis-associated pain: a randomized placebo-controlled trial. Hum Reprod. 2008;23:2017-23.

32. Eisermann J, Gast MJ, Pineda J, Odem RR, Collins JL. Tumor necrosis factor in peritoneal fluid of women undergoing laparoscopic surgery. Fertil Steril. 1988:50:573-9.

33. Braun DP, Gebel H, House R, Rana N, Dmowski NP. Spontaneous and induced synthesis of cytokines by peripheral blood monocytes in patients with endometriosis. Fertil Steril. 1996;65:1125-9.

34. Rana N, Braun DP, House R, Gebel H, Rotman C, Dmowski WP. Basal and stimulated secretion of cytokines by peritoneal macrophages in women with endometriosis. Fertil Steril. 1996:65:925-30.

35. Podgaec S, Dias Junior JA, Chapron C, Oliveira RM, Baracat EC, Abrao MS. Th1 and Th2 ummune responses related to pelvic endometriosis. Rev Assoc Med Bras. 1992;56:92-8.

36. Xavier P, Belo L, Beires J, Rebelo I, Martinez-de-Oliveira J, Lunet N, et al. Serum levels of VEGF and TNF-alpha and their association with C-reactive protein in patients with endometriosis. Arch Gynecol Obstet. 2006;273:227-31.

37. Zhang RJ, Wild RA, Ojago JM. Effect of tumor necrosis factor-alpha on adhesion of human endometrial stromal cells to peritoneal mesothelial cells: an in vitro system. Fertil Steril. 1993;59:1196-201.

38. Bedaiwy MA, Falcone T. Laboratory testing for endometriosis. Clin Chim Acta. 2004:340:41-56.

39. Bertazza L, Mocellin S. The dual role of tumor necrosis factor (TNF) in cancer biology. Curr Med Chem. 2010;17:3337-52.

40. Galo S, Zubor P, Szunyogh N, Kajo K, Machalekova K, Biringer K, et al. TNFalpha serum levels in women with endometriosis: prospective clinical study. Ceska Gynekol. 2005;70:286-90.

41. Zhou J, Ye F, Chen H, Lv W, Gan N. The expression of interleukin-10 in patients with primary ovarian epithelial carcinoma and in ovarian carcinoma cell lines. J Int Med Res. 2007:35:290-300.

42. Berger S, Siegert A, Denkert C, Kobel M, Hauptmann S. Interleukin-10 in serous ovarian carcinoma cell lines. Cancer Immunol Immunother. 2001:50:328-33.

43. Berek JS, Bast Jr RC, Lichtenstein A, Hacker NF, Spina CA, Lagasse LD, et al. Lymphocyte cytotoxicity in the peritoneal cavity and blood of patients with ovarian cancer. Obstet Gynecol. 1984;64:708-14.

44. Mustea A, Konsgen D, Braicu El, Pirvulescu C, Sun P, Sofroni D, et al. Expression of IL-10 in patients with ovarian carcinoma. Anticancer Res. 2006;26:1715-8.

45. Yigit R, Figdor CG, Zusterzeel PL, Pots JM, Torensma R, Massuger LF Cytokine analysis as a tool to understand tumour-host interaction in ovarian cancer. Eur J Cancer. 2011:47:1883-9.

46. Matte I, Lane D, Laplante C, Rancourt C, Piche A. Profiling of cytokines in human epithelial ovarian cancer ascites. Am J Cancer Res. 2012;2:566-80.

47. Bukur J, Jasinski S, Seliger B. The role of classical and non-classical HLA class I antigens in human tumors. Semin Cancer Biol. 2012;22:350-8. 
48. Yoon BS, Kim YT, Kim JW, Kim SH, Kim JH, Kim SW. Expression of human leukocyte antigen-G and its correlation with interleukin-10 expression in cervical carcinoma. Int J Gynaecol Obstet. 2007;98:48-53.

49. Cassatella MA, Meda L, Bonora S, Ceska M, Constantin G. Interleukin 10 (IL-10) inhibits the release of proinflammatory cytokines from human polymorphonuclear leukocytes. Evidence for an autocrine role of tumor necrosis factor and IL-1 beta in mediating the production of IL-8 triggered by lipopolysaccharide. J Exp Med. 1993;178:2207-11.

50. Kanai T, Fujii T, Kozuma S, Yamashita T, Miki A, Kikuchi A, Taketani Y. Soluble HLA-G influences the release of cytokines from allogeneic peripheral blood mononuclear cells in culture. Mol Hum Reprod. 2001;7:195-200.

51. Kleinberg L, Florenes V, Skrede M, Dong H, Nielsen S, McMaster M, et al. Expression of HLA-G in malignant mesothelioma and clinically aggressive breast carcinoma. Virchows Arch. 2006;449:31-9.

52. Ye S, Yang H, Li K, Dong D, Lin X, Yie S. Human leukocyte antigen $G$ expresion: as a significant prognostic indicator for patients with colorectal cancer. Mod Pathol. 2007:20:375-83.

53. Sebti Y, Le Maux A, Gros F, De Guibert S, Pangault C, Rouas-Freiss N, et al. Soluble HLA-G molecules are increased in lymphoproliferative disorders. Br J Hematol. 2007;138:202-12.

54. Gros F, Sebti Y, de Guiberty S, Branger B, Bernard M, Fauchet R, et al. Soluble HLA$G$ molecules are increased during acute leukemia, especially in subtypes affecting monocytic and lymphoid lineages. Neoplasia. 2006;8:223-30.

\section{Submit your next manuscript to BioMed Central and we will help you at every step:}

- We accept pre-submission inquiries

- Our selector tool helps you to find the most relevant journal

- We provide round the clock customer support

- Convenient online submission

- Thorough peer review

- Inclusion in PubMed and all major indexing services

- Maximum visibility for your research

Submit your manuscript at www.biomedcentral.com/submit

C) Biomed Central 